Supporting Information

\title{
Printed Stretchable Single-Nanofiber Interconnections for Individually-Addressable Highly-Integrated Transparent Stretchable Field Effect Transistor Array
}

Dong Wook Kim, Jihye Kwon, Hyoung Seop Kim, and Unyong Jeong*

Department of Materials Science and Engineering, Pohang University of Science and Technology (POSTECH), 77 Cheongam-Ro, Nam-Gu, Pohang, Gyeongbuk 37673, Republic of Korea

*Corresponding author: ujeong@postech.ac.kr

Keywords: stretchable electronics, transparent electronics, printed electronics, metal nanofiber, interconnection 


\section{Experimental Section}

Wavy P4VP NFs electrohydrodynamic (EHD) printing: Before P4VP NFs printing, PDMS collector substrates were prepared by curing the PDMS prepolymer mixture (Sylgard 184, Dow Corning) at $80^{\circ} \mathrm{C}$ for $2 \mathrm{~h}$ in an oven. The prepolymer and the crosslinking agent was 10:1 (w:w) in the mixture. The cured PDMS substrate ( $\sim 60 \mu \mathrm{m}$ thick) was treated with oxygen plasma (100 W, $1 \mathrm{~min})$ to oxidize its surface. After plasma treatment, the PDMS substrate was placed in a chamber with a humidity above $70 \%$, and a vaporized water layer was formed on its hydrophilic surface. For the P4VP NFs printing, $30 \mathrm{wt} \%$ of P4VP $\left(M_{\mathrm{W}}=\sim 160000\right.$, Aldrich $)$ dissolved in $N, N$-dimethyformamide (99.9\%, anhydrous, Aldrich)/ethanol (99.0\%, Samchun) solvent $(60: 40, \mathrm{w}: \mathrm{w})$ was injected through the metal nozzle at $0.1 \mu \mathrm{min}^{-1}$; The nozzle tip-tocollector distance was maintained at $6 \mathrm{~mm}$ during printing. The robotic stage moved in the $\mathrm{X}$ and $\mathrm{Y}$ directions at $650 \mathrm{~mm} \mathrm{~s}^{-1}$ while the NFs were printed on the substrate, and a voltage of $\sim 1.5 \mathrm{kV}$ was applied to the nozzle.

Electroless silver and gold plating of the P4VP NFs: A silver precursor (silver nitrate, $\mathrm{AgNO}_{3}$, Aldrich) was dissolved in deionized water $(25 \mathrm{mg} / \mathrm{ml})$. Printed P4VP NFs template was oxidized by a $10 \mathrm{~min} \mathrm{UV} / \mathrm{O}_{3}$ treatment and immersed in the precursor solution for $1 \mathrm{~min}$ to form complexes with precursor ions. After complexation, the template was dipped in water to remove excess precursor ions and then blown-dried using air. Then, to reduce $\mathrm{Ag}$ precursor to Ag, five drops of diluted hydrazine monohydrate $\left(\mathrm{N}_{2} \mathrm{H}_{4} \cdot \mathrm{H}_{2} \mathrm{O}\right.$, Aldrich) in water $(50 \%, \mathrm{v} / \mathrm{v})$ were dropped into a petri dish, and Ag precursor-complexed template was placed inside of the petri-dish cover for $5 \mathrm{~min}$. Then, the template was dipped in the water to remove residual $\mathrm{N}_{2} \mathrm{H}_{4}$ vapor and blown dry by air. This complexation-reduction cycles were proceeded two times for the Ag plating. For the gold plating of the P4VP NFs, a gold precursor (gold (iii) chloride trihydrate, $\mathrm{HAuCl}_{4} \cdot 3 \mathrm{H}_{2} \mathrm{O}$, Aldrich) was dissolved in deionized water $(0.02 \mathrm{M})$. The template oxidation, complexation, and reduction processes are the same as the silver plating process mentioned above. However, in case of gold plating, the complexation-reduction cycles are repeated three times in total. Water for all experiments was prepared in a Vivagen EXL-3 water purification system and had a resistivity of $>18.2 \mathrm{M} \Omega \mathrm{cm}$.

All-stretchable transparent NF-FET fabrication: DPP NFs printing solution was 
prepared by mixing diketopyrrolopyrrole-thienothiophene (PDPP2T-TT-OD) semiconductor polymer $\left(M_{\mathrm{W}}=40000 \sim 60000, \mathrm{PDI}=2.5 \sim 3.0\right.$, Aldrich $)$ and PEO $\left(M_{\mathrm{W}}=400000\right.$, Aldrich $)$ $(8: 2, \mathrm{w}: \mathrm{w})$ in a co-solvent of the trichloroethylene (Samchun) and chlorobenzene (Aldrich) $(2: 1.3, \mathrm{w}: \mathrm{w})$. The printing solution was injected through the metal nozzle at $0.2 \mu 1 \mathrm{~min}^{-1}$. The nozzle tip-to-collector distance was maintained at $5 \mathrm{~mm}$ during printing. The robotic stage moved at $300 \mathrm{~mm} \mathrm{~s}^{-1}$ and a voltage of $\sim 1.1 \mathrm{kV}$ was applied to the nozzle. The DPP NFs were printed on the pre-strained PDMS substrate with the S/D wavy Au NFs (50 $\mu \mathrm{m}$ spacing). After DPP NFs printing, the pre-strain of the substrate was released, and the wavy DPP NFs were formed. The resulting sample was annealed at $120{ }^{\circ} \mathrm{C}$ for $30 \mathrm{~min}$ to strengthen the contact between the DPP NFs and Au NFs. For the SEBS dielectric layer with the electrospun Ag NFs gate electrodes, SEBS $\left(M_{\mathrm{W}}=118000\right.$, Aldrich) solution (5 wt $\%$ in toluene) was spin-coated on the n-octadecyltrichlorosilane (OTS)-treated glass substrate and annealed at $120{ }^{\circ} \mathrm{C}$ for 30 min. The OTS treatment was necessary for easy detachment of the SEBS layer from the glass substrate. On the SEBS dielectric layer, P4VP NFs mat was formed by electrospinning. The printing solution of the P4VP NFs was injected through the metal nozzle at $0.5 \mu 1 \mathrm{~min}^{-1}$ and the nozzle tip-to-collector distance was maintained at $10 \mathrm{~cm}$ during printing and a voltage of $8.0 \mathrm{kV}$ was applied to the nozzle. The electrospun P4VP NFs were covered with the shadow mask and oxidized by a $10 \mathrm{~min} \mathrm{UV} / \mathrm{O}_{3}$ treatment. The covered non-oxidized P4VP NFs were dissolved out by immersing the sample in ethanol. After P4VP NF patterns formation, the wavy P4VP NFs were printed on the NF patterns for interconnection. The electroless Ag plating of the interconnected P4VP NF patterns was proceeded in the same method mentioned above. Finally, the SEBS layer with the Ag NFs gate electrodes were transferred onto the DPP and Au NFs and annealed at $120^{\circ} \mathrm{C}$ in vacuum for 1 hour.

Finite element method (FEM) simulation: Simulation of the strain distribution in a single wavy fiber was performed using Extended FEM (XFEM) in ABAQUS v6.9 package (Simulia). In the simulation, the total length of the fiber was fixed at $100 \mu \mathrm{m}$, and the fiber was stretched at 10,20,30, and 40\% and held for 4 seconds. The elastic modulus of the P4VP fiber and PDMS substrate was set as 2.5 GPa and $2 \mathrm{MPa}$, respectively, and the Poisson's ratio of the P4VP fiber and PDMS substrate was set as 0.35 and 0.5 , respectively. The periodic boundary condition was applied to the substrate and fiber for an efficient implementation of the continuously repeating fiber structure. 
Characterization: Scanning electron microscope images were observed using a scanning electron microscope (S-4800, Hitachi) at an acceleration voltage of 3-10 kV. Optical microscope images were recorded using an optical microscope (BX51M, Olympus). Transmission electron microscope images and elements mapping images were observed using a high-resolution transmission electron microscope (HR-TEM) equipped with an energy dispersive X-ray spectroscopy (JOEL JEN-2200FS, National Institute for Nanomaterials Technology, Korea). The optical transmittance spectra were obtained using a UV-vis spectrophotometer (S-3100, Scinco). Fourier transform infrared spectroscopy was performed in ATR mode (Vertex 70v, Bruker). Electrical measurements and stretchability test were conducted using a universal measurement probe system (Tera Leader). I- $V$ characteristic measurement were conducted using a parameter analyzer (Keithley 4200) in a $\mathrm{N}_{2}$ atmosphere at room temperature. Sheet resistance was measured using a four point probe (CMT-100S, Advanced Instrument Technology). 

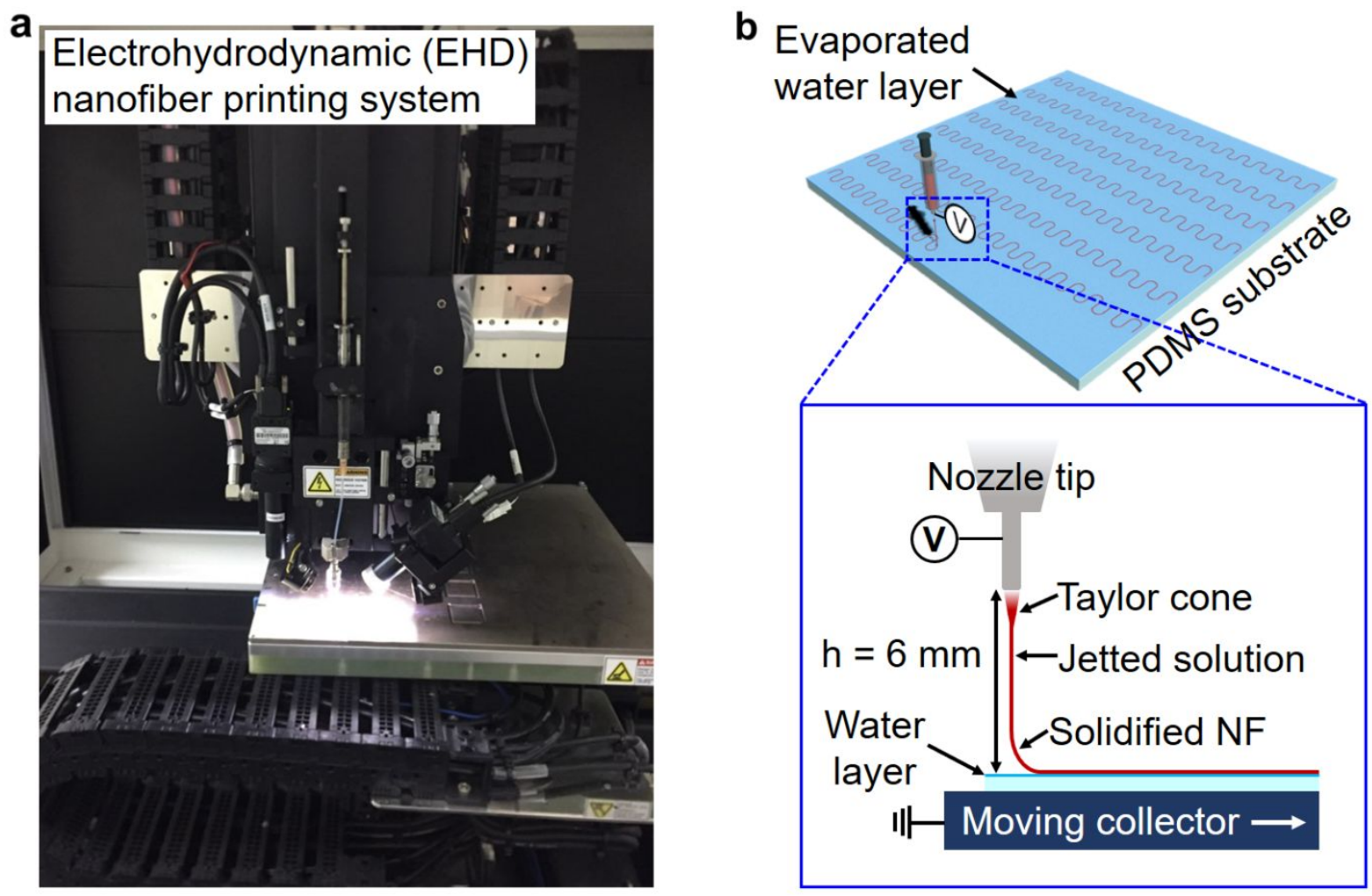

Figure S1. (a) Photograph of the home-built motorized electrohydrodynamic (EHD) printing system. (b) Schematic diagram of the EHD nanofiber (NF) printing on the PDMS substrate with an evaporated water layer. 


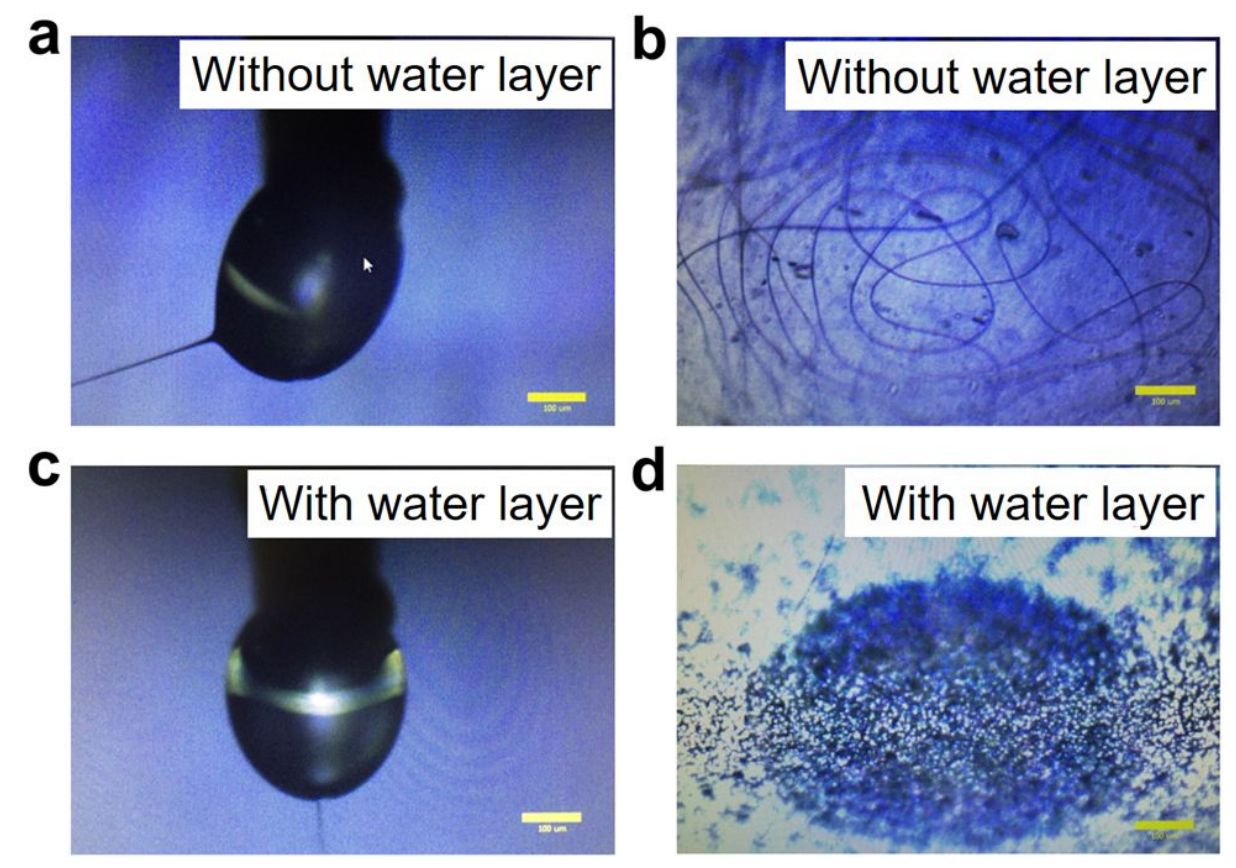

Figure S2. (a-d) OM images of the ejected P4VP jets and the deposited P4VP NFs without (a, b) and with (c, d) an evaporated water layer on the PDMS substrate (scale bar : $100 \mu \mathrm{m}$ ). 

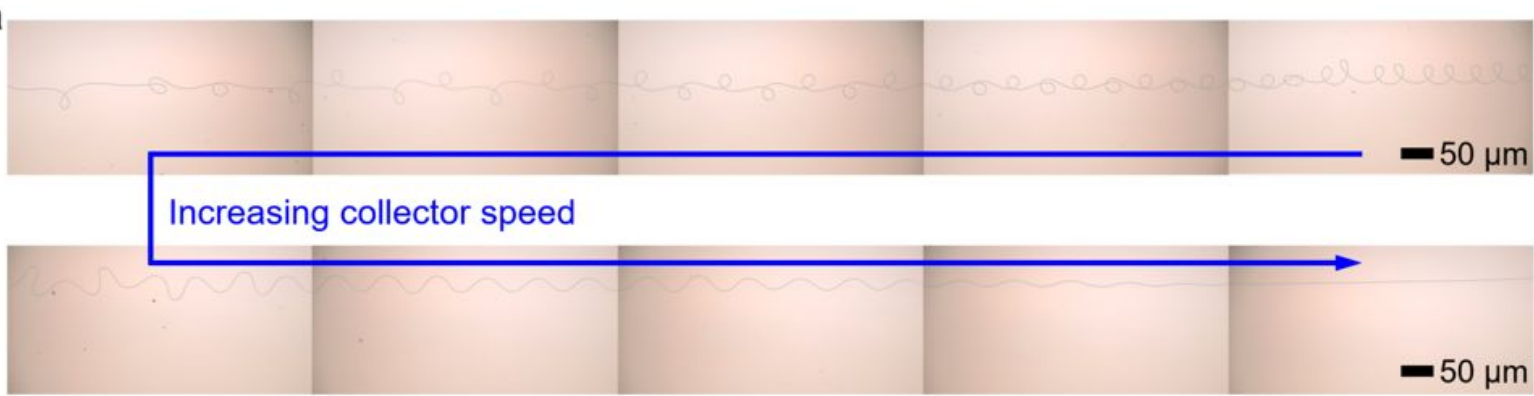

b Collector speed $400 \mathrm{~mm} / \mathrm{s}$

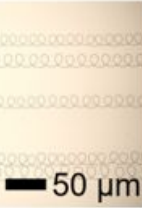

$550 \mathrm{~mm} / \mathrm{s}$

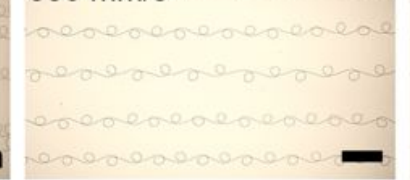

$650 \mathrm{~mm} / \mathrm{s}$

C

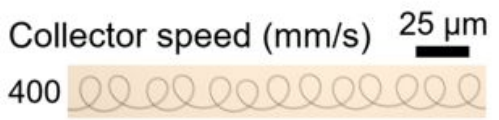

550

600

650

700

750

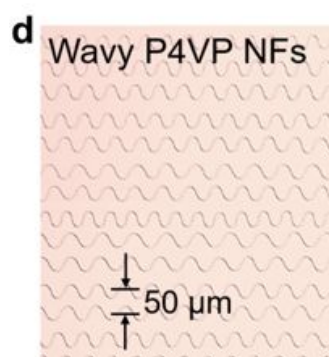

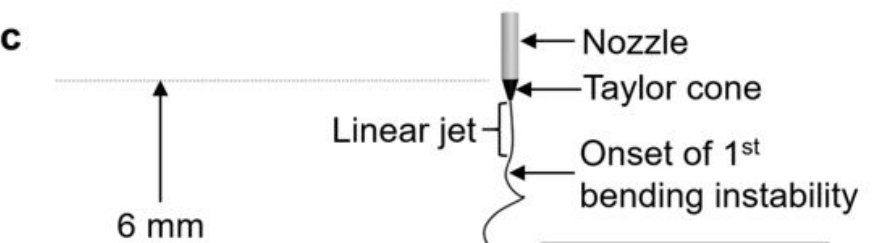

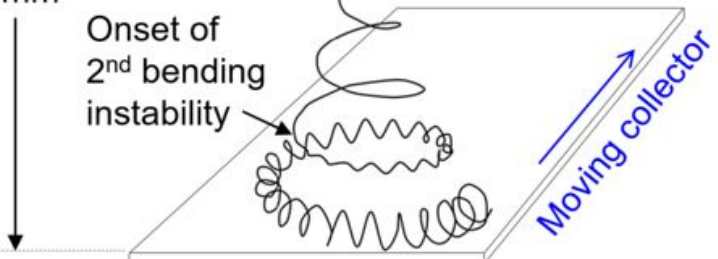

e Grid pattern P4VP NFs $50 \mu \mathrm{m}$

Figure S3. (a) OM images showing the shape change of the P4VP NF with increasing collector (printing) speed. Total $10 \mathrm{OM}$ images were combined to clearly show the shape change. (b) OM images of the P4VP NFs printed at different collector speed. (c) Schematic diagram showing the instantaneous path of an EHD printed jet containing successive electrical bending instabilities. Bending instability of a polymer jet creates a 3D coil structure as it takes flight toward a collector surface. (d, e) OM images of the wavy P4VP NFs array EHD printed with parallel (d) and grid patterns (e). 

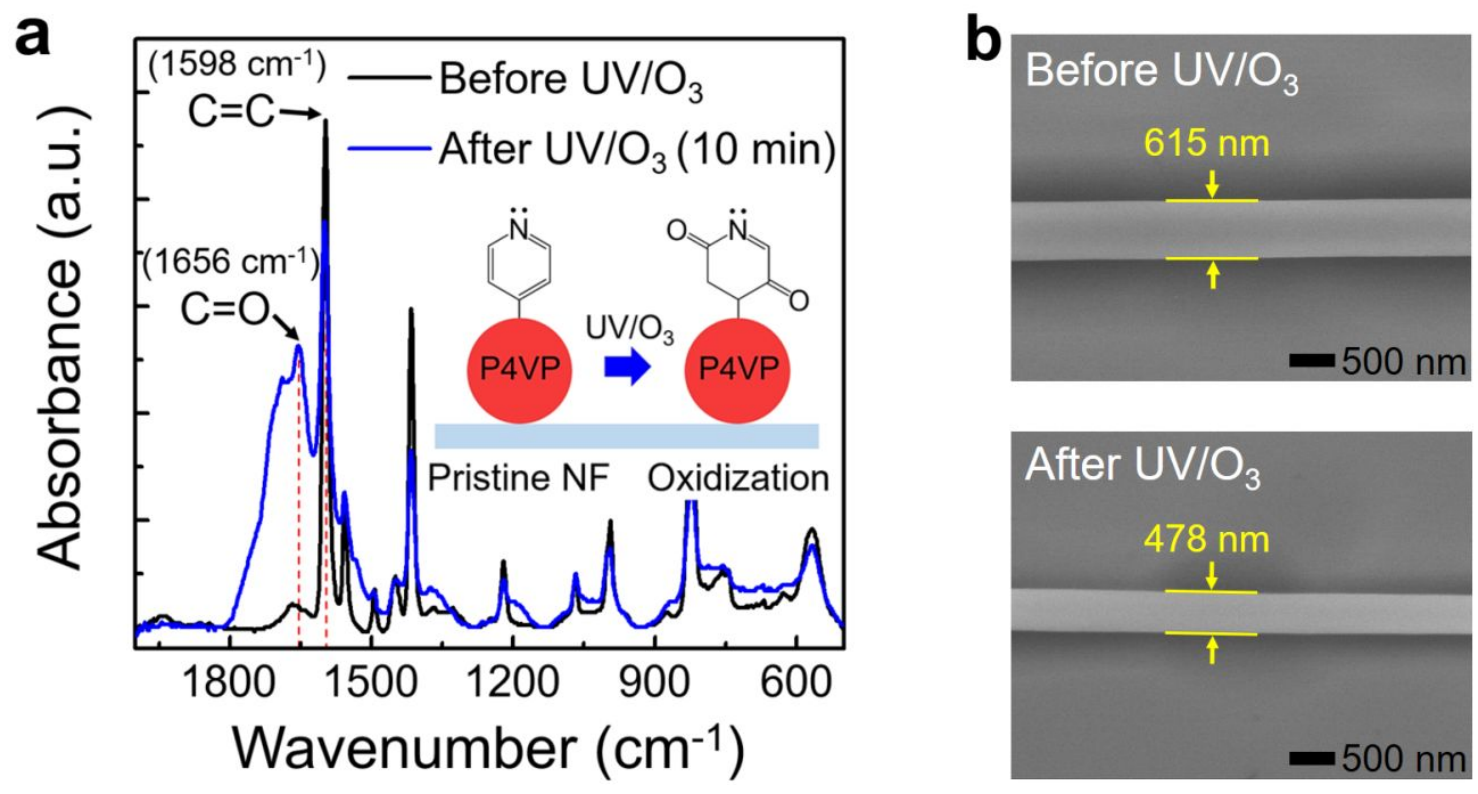

Figure S4. (a) FT-IR absorbance spectra of the P4VP NFs before and after UV/O 3 treatment for 10 min (inset : scheme of molecular change in $\mathrm{P} 4 \mathrm{VP}$ by $\mathrm{UV} / \mathrm{O}_{3}$ treatment) (b) SEM images of the pristine $\mathrm{P} 4 \mathrm{VP} \mathrm{NF}$ and the $\mathrm{UV} / \mathrm{O}_{3}$-treated $\mathrm{P} 4 \mathrm{VP} \mathrm{NF}$. 

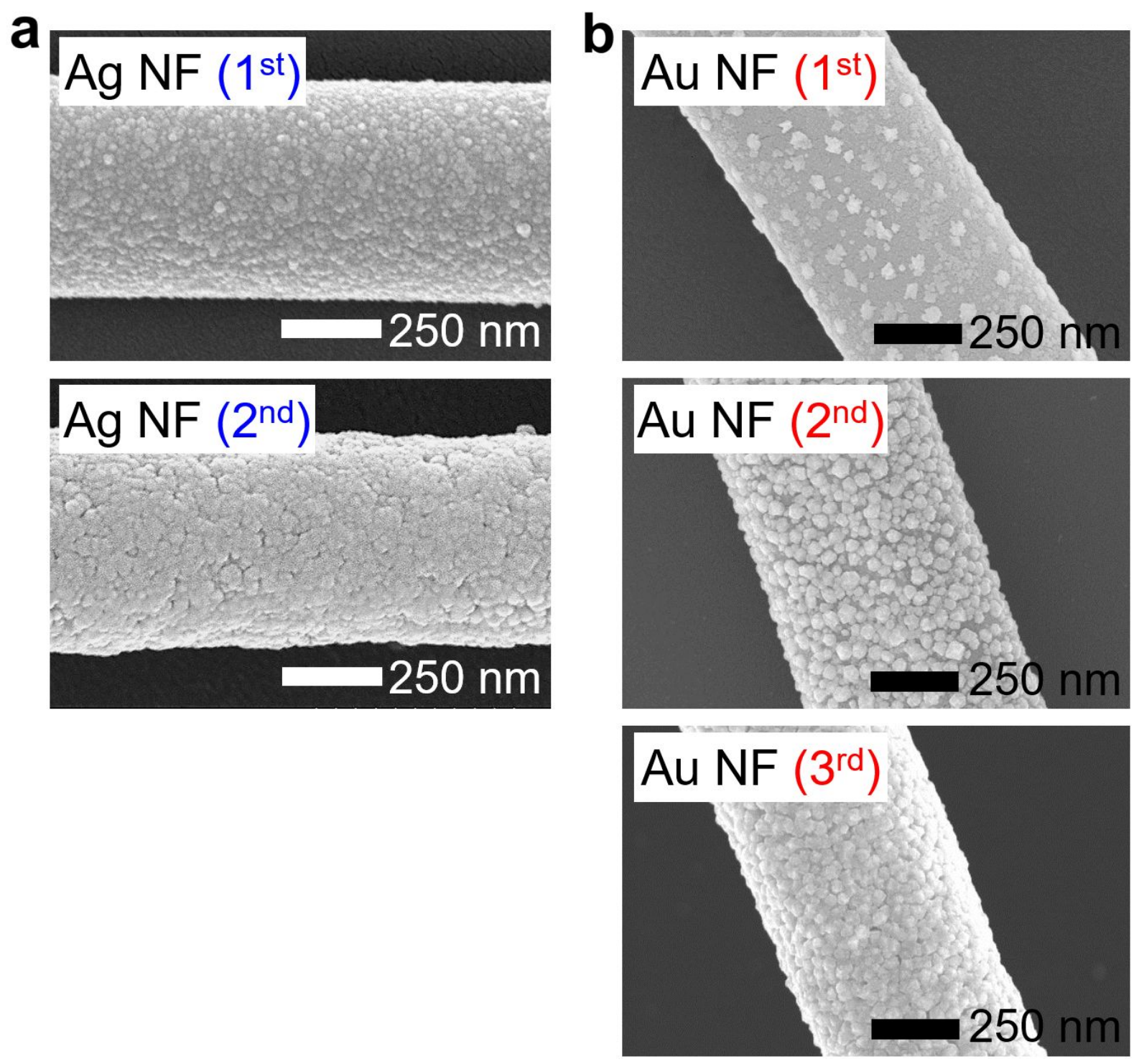

Figure S5. (a-b) SEM images showing the increase in the density of the Ag (a) and Au (b) NPs on the P4VP NF surfaces over the repeated complexation-reduction cycles. 

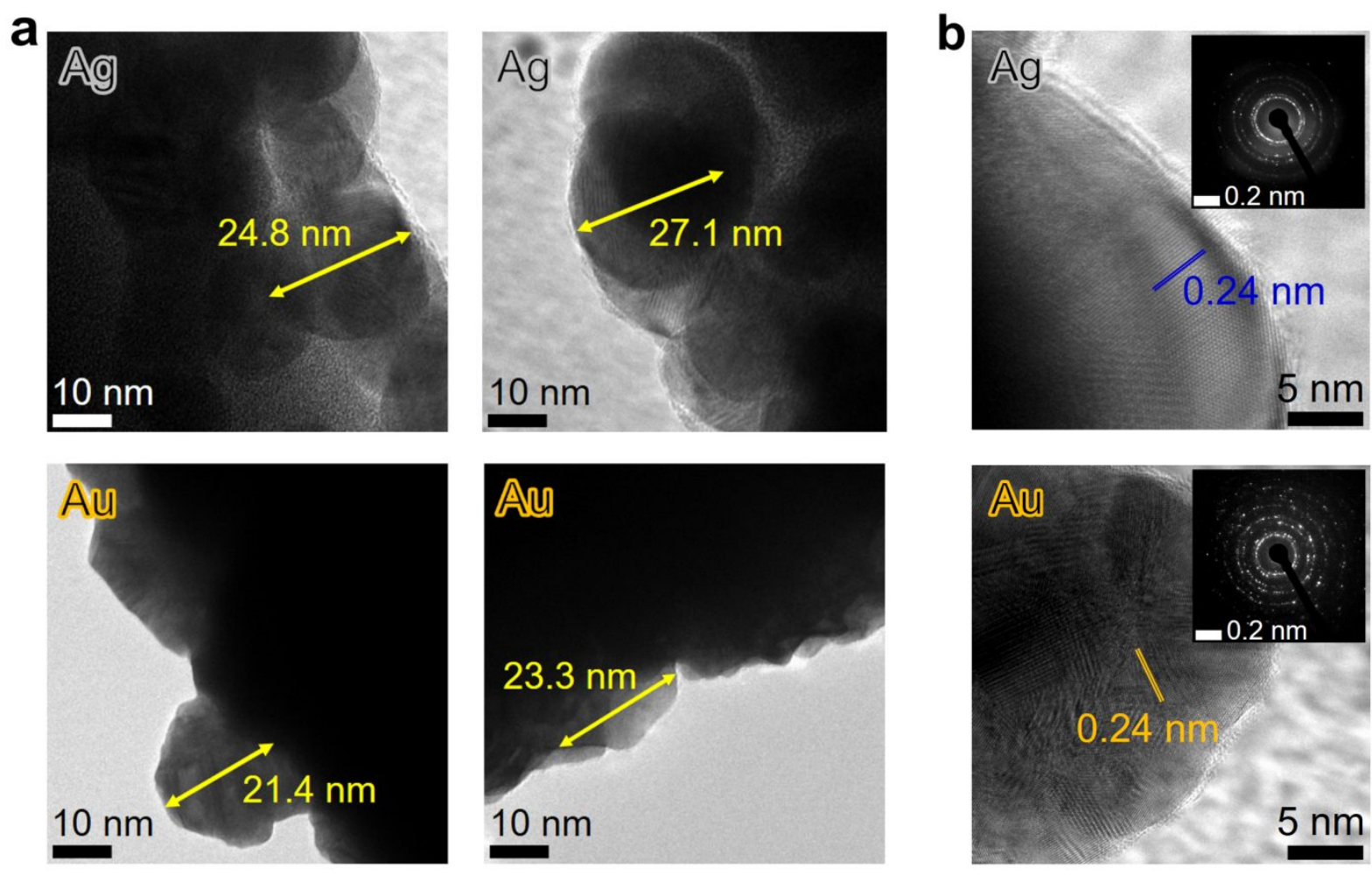

Figure S6. (a) TEM images of the Ag and Au NFs showing the size and shape of the metal NPs on the P4VP NF surfaces. (b) High resolution TEM images of the Ag and Au NPs showing the lattice fringes and the polycrystalline diffraction patterns. 


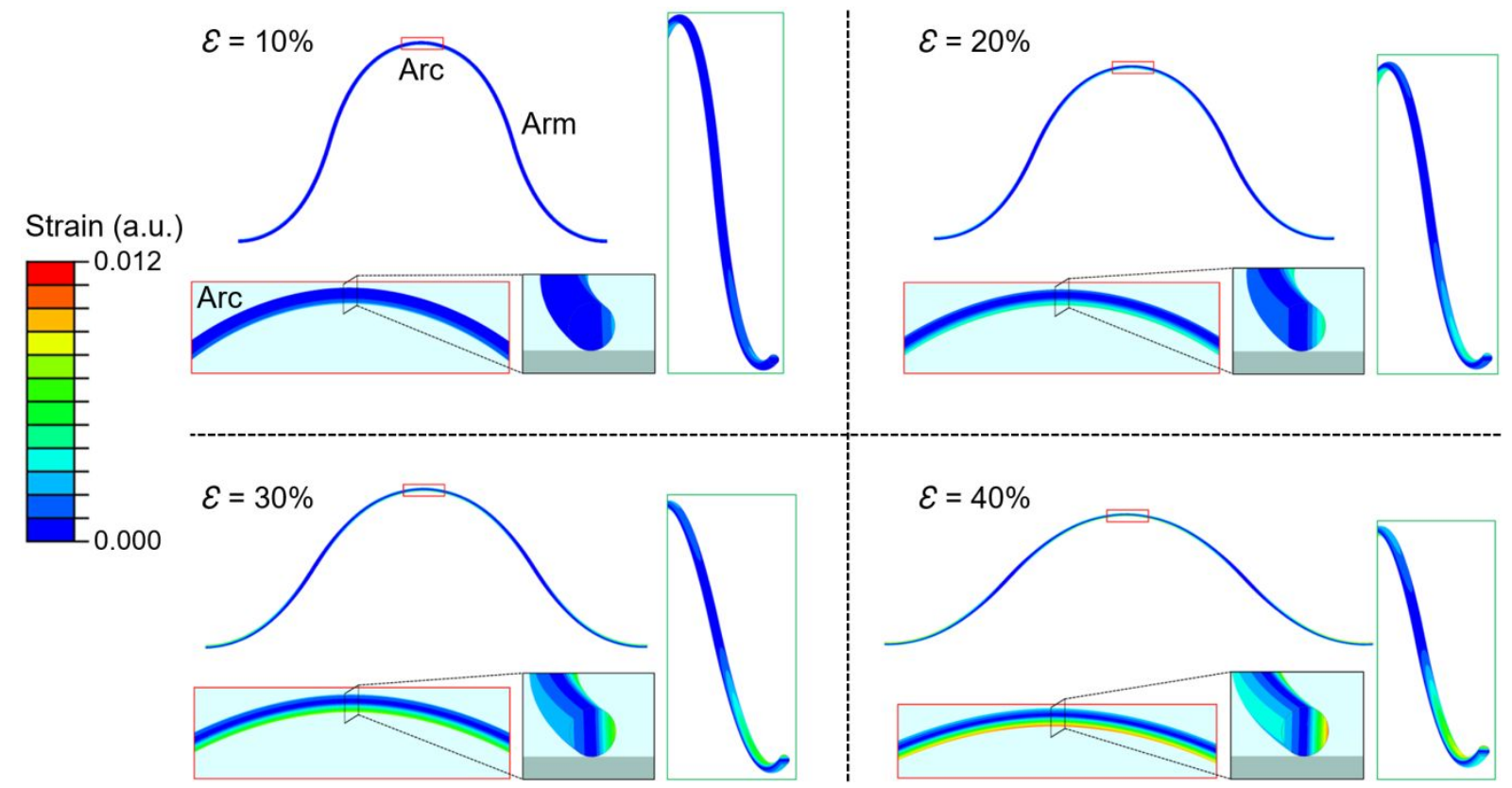

Figure S7. Finite element method (FEM) modeling of the strain distribution in the wavy NF at different uniaxial strains. 

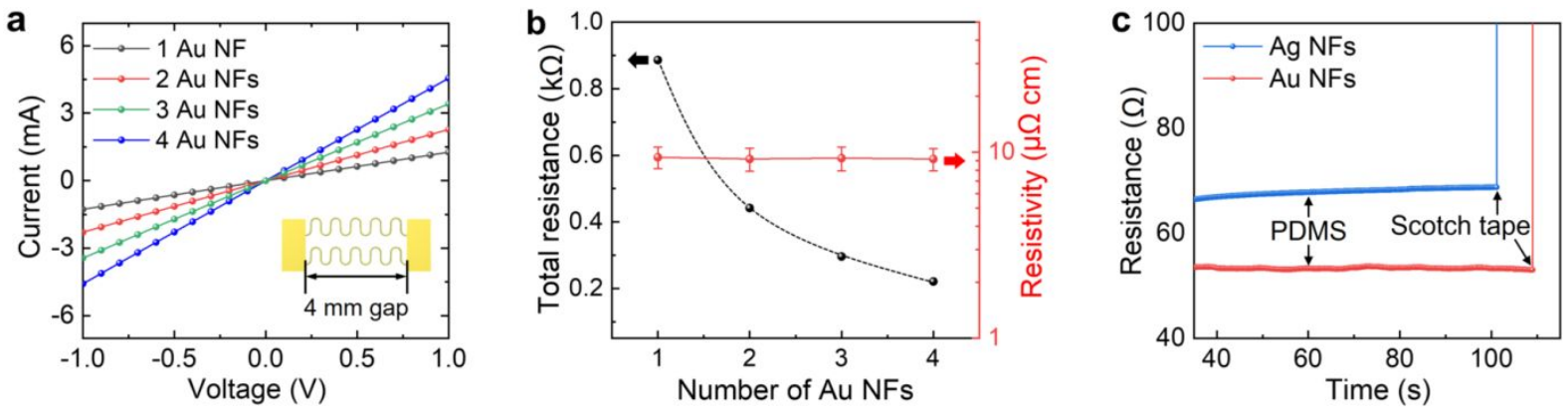

Figure S8. (a) Current-voltage curves and (b) total resistance and resistivity of the wavy Au NFs with different number of fibers. (c) Resistance change of the $10 \mathrm{Ag}$ NFs and $10 \mathrm{Au}$ NFs array during peeling tests using a sticky PDMS film and a scotch tape. The metal NFs were not peeled off by a sticky PDMS film and remained conductive. However, when the NFs were peeled off with a scotch tape, they lost conductivity. 


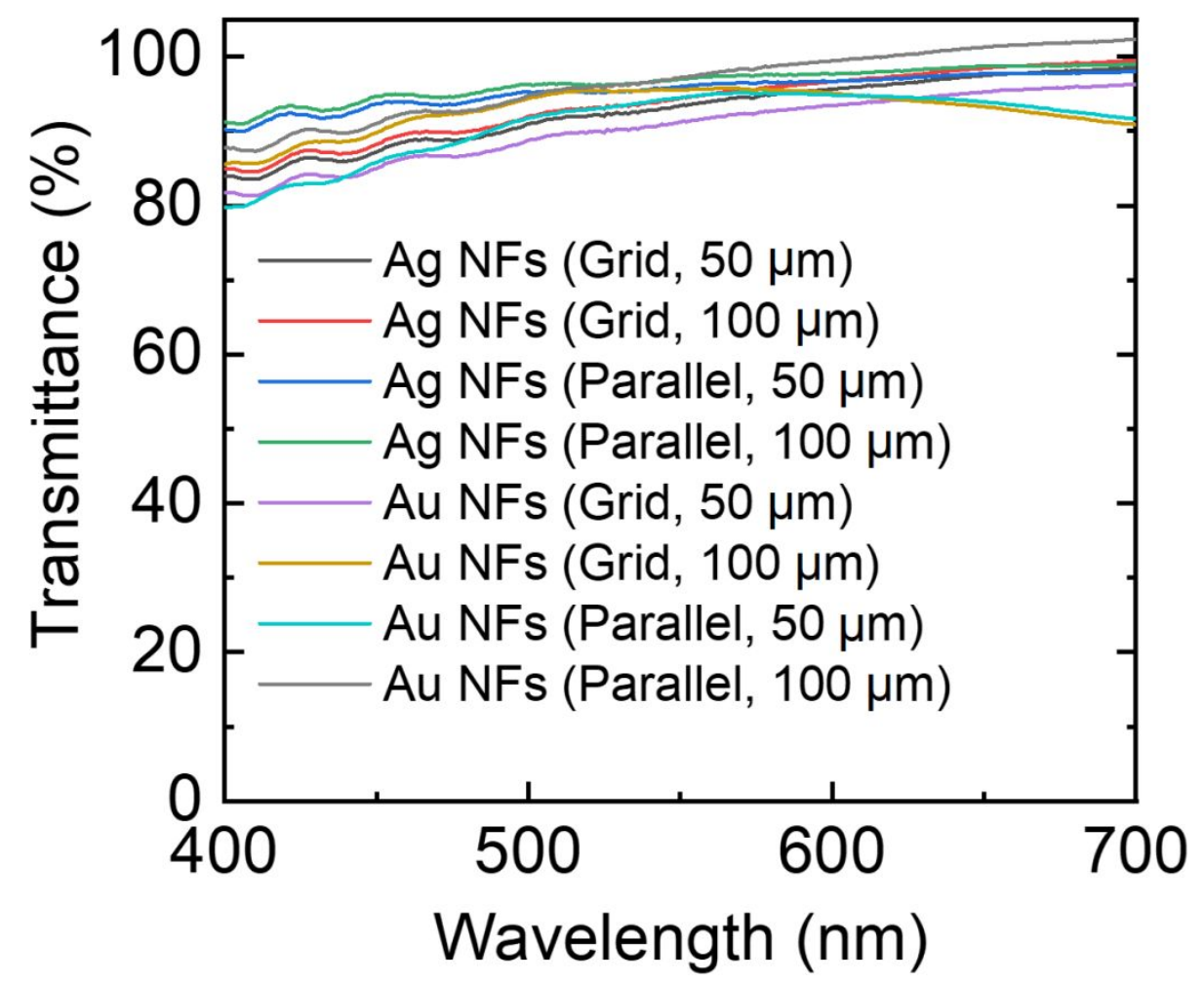

Figure S9. Transmittance of the wavy Ag and Au NFs array with different alignments (grid and parallel) and fiber-spacing $(50 \mu \mathrm{m}$ and $100 \mu \mathrm{m})$. 

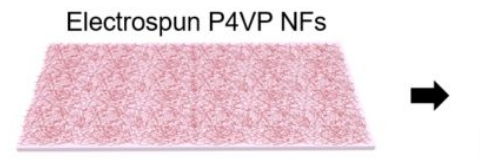

Selective UV/O 3 patterning $\downarrow \downarrow \downarrow \downarrow \downarrow \downarrow \downarrow \downarrow \downarrow \downarrow$

Interconnected Ag NFs patterns (G)

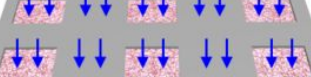

$\downarrow$

Wavy P4VP NFs EHD printing

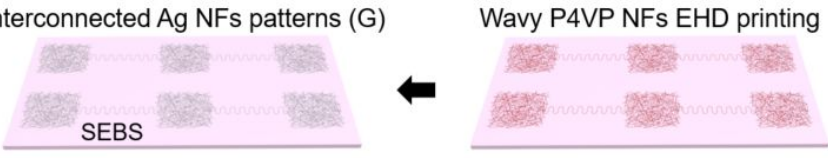

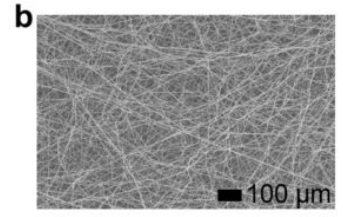

Electrospun Ag NF (1st)

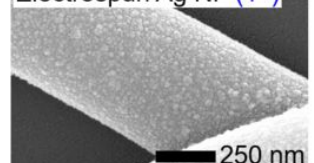

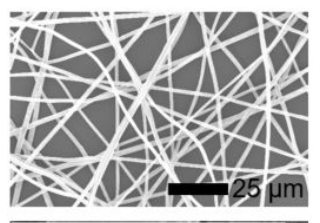

Electrospun $\mathrm{Ag} \mathrm{NF}\left(2^{\text {nd }}\right)$
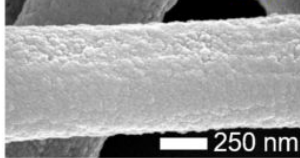

Figure S10. (a) Schematic showing the fabrication process of the SEBS (dielectric layer) / interconnected Ag NFs patterns (gate electrodes, G). (b) SEM images of the electrospun Ag NFs and the NF surfaces over the repeated Ag ions complexation-reduction cycles. 


\section{a Wavy DPP NFs}

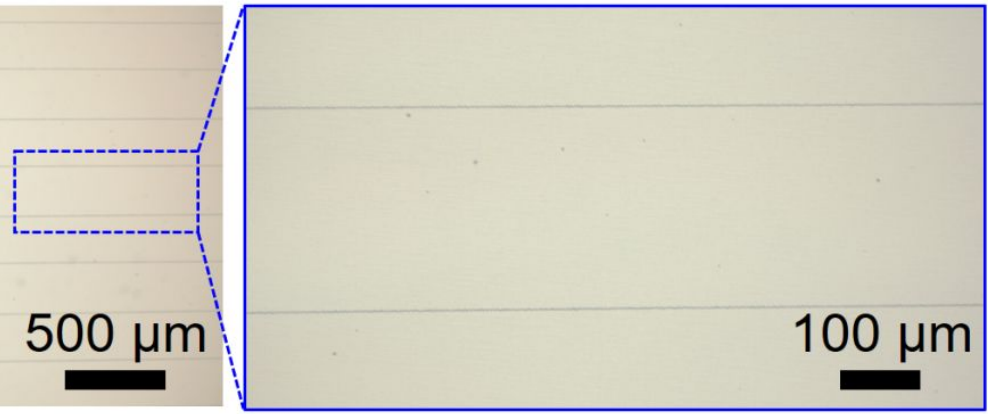

b Before taping

\section{After taping}
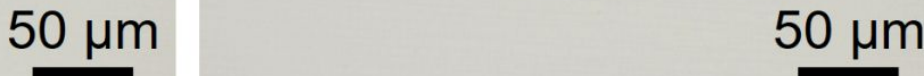

Figure S11. (a) OM images of the printed wavy DPP NFs on the PDMS substrate. (b) OM images of the DPP NF before and after peeling off using a scotch tape. 
a

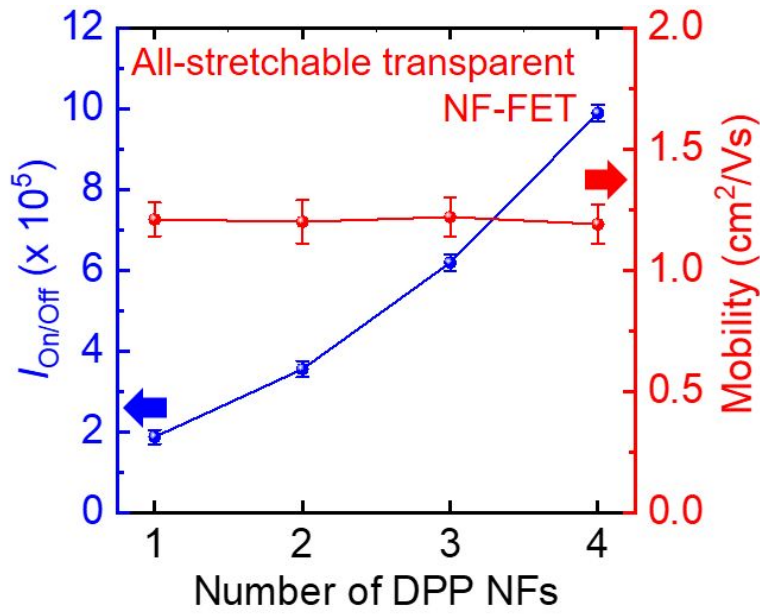

C

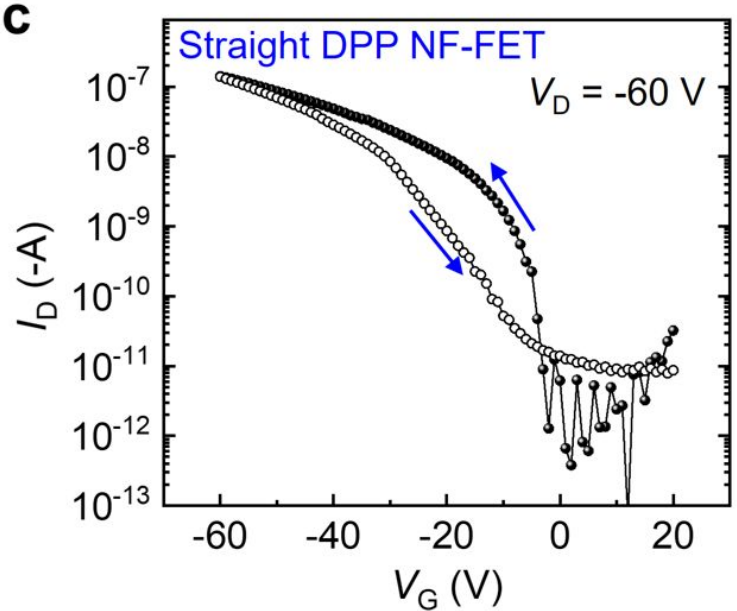

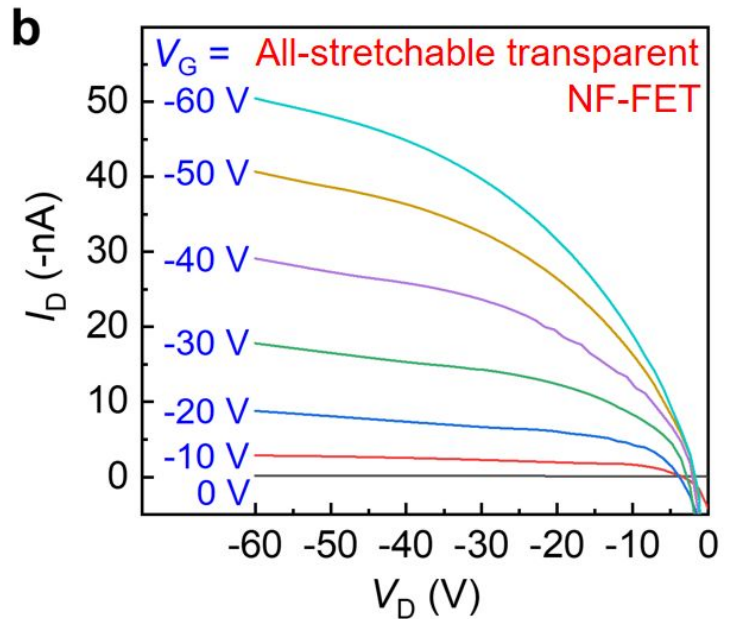

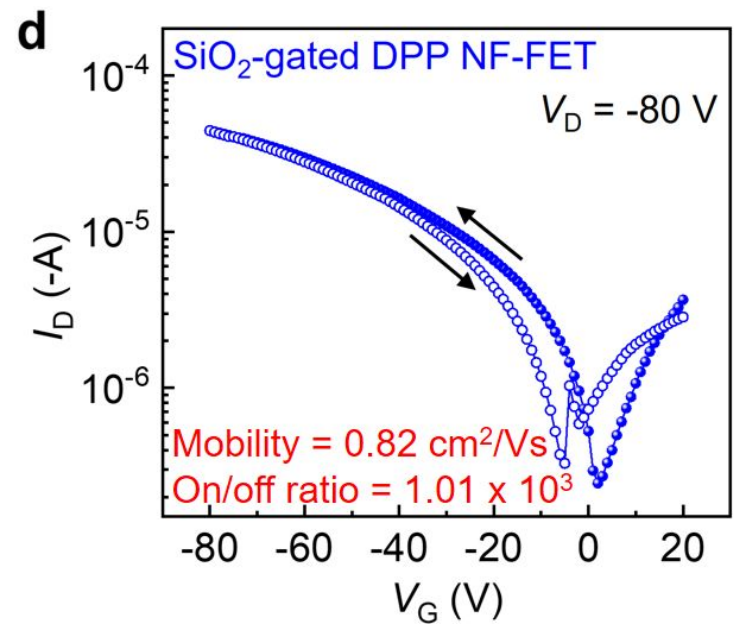

Figure S12. (a) On/off ratio and hole mobility versus the number of DPP NFs. (b) Output curves of the all-stretchable transparent NF-FET. (c) Transfer curve of the NF-FET with the straight DPP NFs. The wavy Au NFs (S/D), SEBS dielectric layer, and Ag NFs (G) of the allstretchable transparent NF-FET were used same for the measurement. (d) Transfer curve of the $\mathrm{SiO}_{2}$-gated DPP NF-FET. DPP NFs were printed on the $\mathrm{SiO}_{2}(300 \mathrm{~nm}) / \mathrm{Si}$ wafer and the wavy $\mathrm{Au}$ NFs were used as S/D electrodes (bottom-gate bottom-contact configuration). 

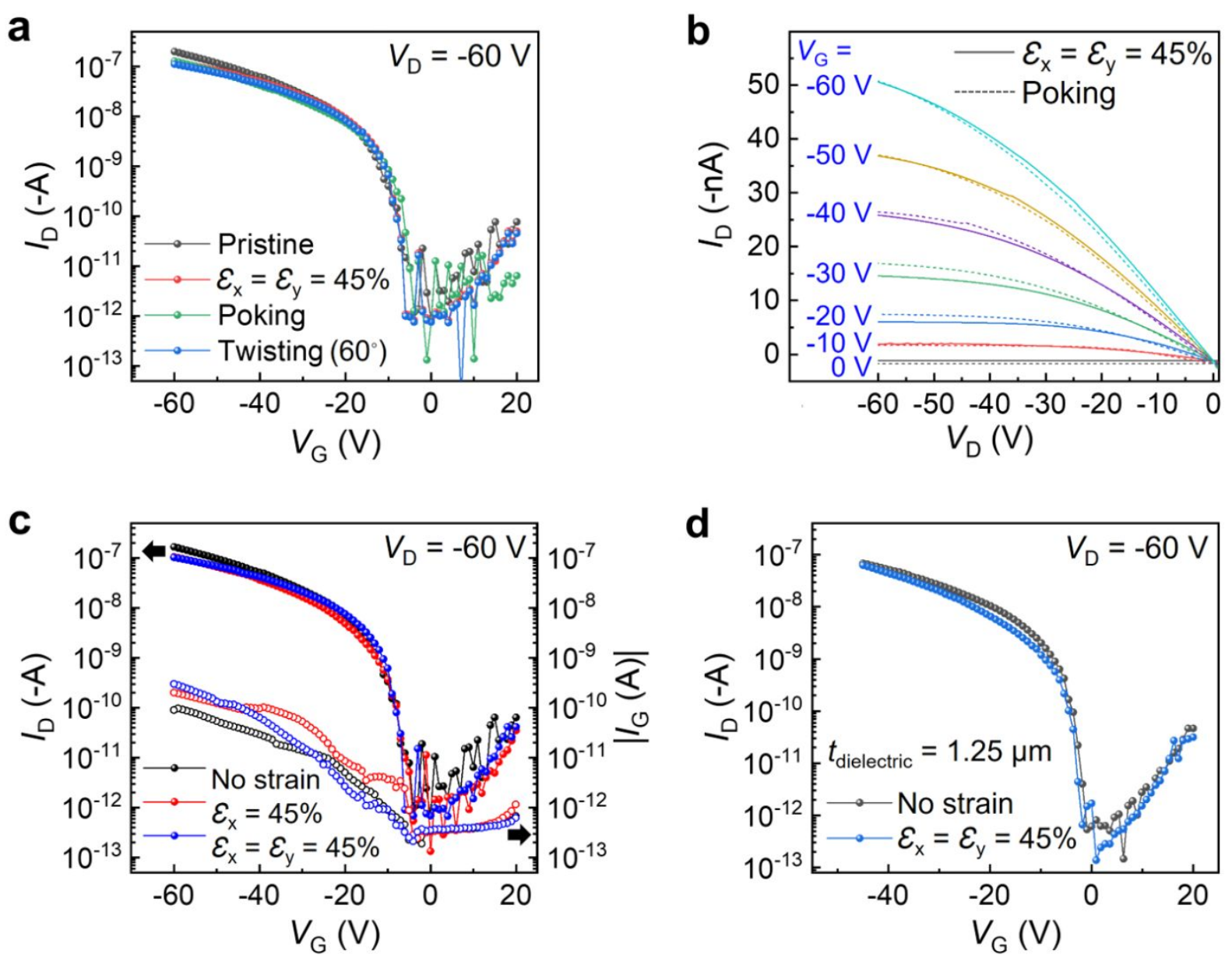

Figure S13. Transfer curves of the NF-FET under various mechanical deformations. (b) Output curves of the NF-FET at biaxially stretching (solid lines) and poking states (dashed lines). (c) Drain current $\left(I_{\mathrm{D}}\right)$ and gate leakage current $\left(I_{\mathrm{G}}\right)$ of the NF-FET at different strains and strain directions. (d) Transfer curves of the NF-FET with a $1.25 \mu \mathrm{m}$-thick SEBS dielectric layer. The transfer curves were obtained at pristine and biaxially stretched states. 